\title{
MODEL-INDEPENDENT ESTIMATORS FOR THE STATISTICAL ANISOTROPY OF THE COSMIC MICROWAVE BACKGROUND
}

\author{
YU.V. SHTANOV, D.O. SAVCHENKO
}

PACS 98.80.Cq; 98.70.Vc;

Bogolyubov Institute for Theoretical Physics, Nat. Acad. of Sci. of Ukraine

(C) 2011

(14b, Metrologichna Str., Kyiv 03680, Ukraine; e-mail: shtanov@bitp. kiev.ua)

\begin{abstract}
We construct estimators for testing the statistical anisotropy of the cosmic microwave background arising due to a quadrupole scale-independent anisotropy in the primordial power spectrum. The estimators do not require the knowledge of basic cosmological parameters. We determine the sensitivity of the constructed estimators to the magnitude of the statistical anisotropy and perform test simulations that confirm our theoretical estimates.
\end{abstract}

\section{Introduction}

The observable Universe is only approximately homogeneous and isotropic. Departures from homogeneity and isotropy are small in the past and/or on large spatial scales and are detected in the observations of the largescale structure and the cosmic microwave background (CMB). Thus, the CMB temperature $T(\mathbf{n})$ slightly depends on the unit vector $\mathbf{n}$ indicating the direction of observation, with characteristic variation $\Delta T / T_{0} \sim 10^{-5}$ with respect to the average temperature $T_{0}$.

Of special interest as of the recent time are the statistical properties of perturbations in the early Universe which may be reflected in the CMB temperature field. Here, the main object of investigation is the correlation function

$C\left(\mathbf{n}_{1}, \mathbf{n}_{2}\right)=\left\langle\Delta T\left(\mathbf{n}_{1}\right) \Delta T\left(\mathbf{n}_{2}\right)\right\rangle$

where $\Delta T(\mathbf{n})=T(\mathbf{n})-T_{0}$. The statistical isotropy of primordial density perturbations is a general assumption of the models of structure formation, leading to the isotropy of the correlation function (1), i.e., its invariance with respect to rotations of the celestial sphere. In this case, it is a function only of the angle between the directions $\mathbf{n}_{1}$ and $\mathbf{n}_{2}: C\left(\mathbf{n}_{1}, \mathbf{n}_{2}\right)=f\left(\mathbf{n}_{1} \cdot \mathbf{n}_{2}\right)$.

Statistical isotropy is one of the robust predictions of the inflationary theory [1] of the origin of primordial perturbations. It is very important to test this prediction by observations. A number of researches recently put this principle to theoretical and experimental tests. On the theoretical side, the issue of the statistical (an)isotropy of the CMB temperature and polarization correlation functions was discussed in a general statement in [2-4] (see a review in [5]). In addition, a number of models were constructed that violate statistical isotropy. These are typically Bianchi I models explicitly breaking the isotropy of the inflationary Universe and driven by various mechanisms: genuine anisotropic initial conditions [6], special uniform vector $[7,8]$ or spinor [9] fields that pick up a preferred direction, anisotropic equation of state [10], or gradients of the scalar fields [11]. More exotic theories can be found in [12].

On the experimental side, two indications of the statistical anisotropy in the CMB temperature map were reported. In one of them, an anisotropy of the quadrupole type in the primordial power spectrum was tested with positive detection [13], which, however, according to the authors, may be not of the cosmological origin, but most likely a product of an unknown systematic effect. Another type of anisotropy is a dipole modulation in the angular power spectrum of the CMB temperature anisotropy, producing slightly different temperature fluctuation powers in the northern and southern celestial hemispheres $[14,15]$. The nature and the origin of this effect still remain unclear; some attempts to explain it are made in [16].

A general form of the anisotropic power spectrum can be presented as

$P_{\mathbf{k}}=P_{k}\left[1+\sum_{L M} g_{L M}(k) Y_{L M}(\hat{\mathbf{k}})\right]$,

where $P_{k}$ is the isotropic part of the power spectrum $P_{\mathbf{k}}$, and $g_{L M}(k)$ are the coefficients of the expansion of the anisotropic part in spherical harmonics $Y_{L M}(\hat{\mathbf{k}})$ (here, $\hat{\mathbf{k}}=\mathbf{k} / k)$. The general statistics for the CMB temperature corresponding to such primordial power spectra was investigated in [4]. In some special cases, such as, e.g., that considered in [7], the sum in (2) contains only har- 
monics with $L=2$, with the coefficients $g_{2 M}$ that do not depend on $k$.

In the case of constant $g_{L M}$, the statistical analysis allows for a great simplification. The aim of this paper is to construct a set of estimators for the constant coefficients $g_{L M}$ in (2) that would be independent of the cosmological parameters such as the densities of various components filling the Universe, equation of state for the dark energy, etc. We then study the statistical properties of such estimators for $g_{2 M}$ in a special case of an anisotropy of the quadrupole type (with $g_{L M}$ nonzero only for $L=2$ ).

\section{Correlation Function for the CMB Temperature}

The temperature map $T(\mathbf{n})$ can be expanded in terms of spherical harmonics $Y_{l m}(\mathbf{n})$ as follows:

$T(\mathbf{n})=T_{0} \sum_{l m} a_{l m} Y_{l m}(\mathbf{n})$.

The covariance matrix for the coefficients $a_{l m}$ is given by [4]

$\left\langle a_{l m} a_{l^{\prime} m^{\prime}}^{*}\right\rangle=\delta_{l l^{\prime}} \delta_{m m^{\prime}} C_{l}+\sum_{L M} \xi_{l m l^{\prime} m^{\prime}}^{L M} D_{l l^{\prime}}^{L M}$

Here,

$$
\begin{aligned}
& \xi_{l m l^{\prime} m^{\prime}}^{L M}=\int Y_{l m}^{*}(\mathbf{n}) Y_{l^{\prime} m^{\prime}}(\mathbf{n}) Y_{L M}(\mathbf{n}) d \mathbf{n}= \\
& =(-1)^{m^{\prime}}\left(G_{l l^{\prime}}^{L}\right)^{1 / 2} C_{l m l^{\prime}-m^{\prime}}^{L M}
\end{aligned}
$$

and

$G_{l l^{\prime}}^{L} \equiv \frac{(2 l+1)\left(2 l^{\prime}+1\right)}{4 \pi(2 L+1)}\left(C_{l 0 l^{\prime} 0}^{L 0}\right)^{2}$,

where $C_{l m l^{\prime} m^{\prime}}^{L M}$ are Clebsch-Gordan coefficients. The quantities $C_{l}$ describe the usual isotropic part of the angular power spectrum and are given by

$C_{l}=(4 \pi)^{2} \int_{0}^{\infty} d k k^{2} P_{k}\left[\Theta_{l}(k)\right]^{2}$.

The anisotropic part of the angular power spectrum is encoded in the quantities $D_{l l^{\prime}}^{L M}$ in (4), given by

$D_{l l^{\prime}}^{L M}=(4 \pi)^{2}(-\mathrm{i})^{l-l^{\prime}} \int_{0}^{\infty} d k k^{2} P_{k} \Theta_{l}(k) \Theta_{l^{\prime}}(k) g_{L M}(k)$.
Here, $\Theta_{l}(k)$ is the contribution to the $l^{\text {th }}$ temperature moment from the wave number $k$, which depends on the parameters of the cosmological model.

If the coefficients $g_{L M}$ are $k$-independent, then, obviously,

$D_{l l^{\prime}}^{L M}=g_{L M} F_{l l^{\prime}}$

where $F_{l l^{\prime}}$ can be read-off from (8). In particular,

$F_{l l}=C_{l}$.

\section{Proposal for Estimators}

A detailed analysis of the CMB statistics with the anisotropic spectrum of the form described in the previous section can be found in [4]. The estimators for the constant parameters $g_{L M}$ constructed therein involve the quantities $C_{l}$ or $F_{l l^{\prime}}$ that depend on the parameters of the isotropic cosmological model (such as the index of the power spectrum, parameters $\Omega$, etc.) through the quantities $P_{k}$ and $\Theta_{l}(k)$ in (7) or (8). The purpose of this paper is to propose model-independent estimators for the parameters $g_{L M}$ and to study their statistics in the case of quadrupole anisotropy $(L=2)$.

For this purpose, consider Eq. (4) with constant $g_{L M}$ for $l=l^{\prime}$ :

$\left\langle a_{l m} a_{l m^{\prime}}^{*}\right\rangle=C_{l}\left(\delta_{m m^{\prime}}+\sum_{L M} \xi_{l m l m^{\prime}}^{L M} g_{L M}\right)$.

All the quantities in this expression can be replaced by estimators obtained directly from observations. Indeed, the average $\left\langle a_{l m} a_{l m^{\prime}}^{*}\right\rangle$ is simply estimated by the product $a_{l m} a_{l m^{\prime}}^{*}$, and the quantity $C_{l}$ is naturally estimated by

$\hat{C}_{l}=\frac{1}{2 l+1} \sum_{m}\left|a_{l m}\right|^{2}$,

since the equality $\left\langle\hat{C}_{l}\right\rangle=C_{l}$ follows from (4) and from the summation properties of the geometrical coefficients (5). Hence, one can consider the collection

$\zeta_{l m m^{\prime}}=a_{l m} a_{l m^{\prime}}^{*}-\hat{C}_{l}\left(\delta_{m m^{\prime}}+\sum_{L M} \xi_{l m l m^{\prime}}^{L M} g_{L M}\right)$

as a set of test functions to look for the parameters $g_{L M}$. Specifically, the "true" collection of the numbers $\left\{g_{L M}\right\}$ should minimize a deviation of the collection of the quantities $\left\{\zeta_{l m m^{\prime}}\right\}$ from zero values.

The above proposal is quite generic and can be used to determine all coefficients $g_{L M}$. If the statistical 
anisotropy is restricted to a quadrupole $(L=2)$, then there are five real constants to be found in $g_{2 M}$ (one real $g_{20}$ and two complex $g_{21}$ and $g_{22}$ ). In this case, the procedure for determining $g_{2 M}$ can be further simplified as follows.

The coefficients $\xi_{l m l m^{\prime}}^{2 M}$ are nonzero only for $\left|m^{\prime}-m\right| \leq$ 2. They are given by the expressions

$\xi_{l m l m^{\prime}}^{2 M}=\xi_{l m}^{M} \delta_{m, m^{\prime}+M}=\xi_{l m}^{M} \delta_{m^{\prime}, m-M}$,

where

$\xi_{l m}^{-2}=(-1)^{l} \sqrt{\frac{15}{8 \pi}} \times$

$\times \frac{\sqrt{(l-m)(l-m-1)(l+m+1)(l+m+2)}}{(2 l-1)(2 l+3)}$,

$\xi_{l m}^{-1}=(-1)^{l} \sqrt{\frac{15}{8 \pi}} \frac{(1+2 m) \sqrt{(l-m)(l+m+1)}}{(2 l-1)(2 l+3)}$,

$\xi_{l m}^{0}=-(-1)^{l} \sqrt{\frac{5}{4 \pi}} \frac{l(l+1)-3 m^{2}}{(2 l-1)(2 l+3)}$

$\xi_{l m}^{1}=(-1)^{l} \sqrt{\frac{15}{8 \pi}} \frac{(1-2 m) \sqrt{(l+m)(l-m+1)}}{(2 l-1)(2 l+3)}$,

$\xi_{l m}^{2}=(-1)^{l} \sqrt{\frac{15}{8 \pi}} \times$

$\times \frac{\sqrt{(l+m-1)(l+m)(l-m+1)(l-m+2)}}{(2 l-1)(2 l+3)}$,

One can see that, for given $l$, all these quantities are bounded by a constant $\mathcal{O}(l)$.

For definiteness, consider the procedure for determining $g_{22}$. Let us consider the functions of a complex parameter $z$

$\zeta_{l m}(z) \equiv(-1)^{l} l(l+1) \zeta_{l m, m-2}=$

$=(-1)^{l} l(l+1)\left(a_{l m} a_{l, m-2}^{*}-z \xi_{l m}^{2} \hat{C}_{l}\right)$.

The statistical average of (20) is

$\left\langle\zeta_{l m}(z)\right\rangle=(-1)^{l} \xi_{l m}^{2} S_{l}\left(g_{22}-z\right)$, turning to zero only for $z=g_{22}$. We have introduced the quantities $S_{l}=l(l+1) C_{l}$, which (up to a coefficient) are the canonical representation for the CMB angular power spectrum. All the quantities $S_{l}$ have comparable order of magnitude. For $z=0$ (which presupposes the statistical isotropy), we have

$\left\langle\zeta_{l m}(0)\right\rangle=(-1)^{l} \xi_{l m}^{2} S_{l} g_{22}$.

Note that the product $(-1)^{l} \xi_{l m}^{2}$ is always positive, as well as $\hat{C}_{l}$ and $C_{l}$. It is for this positiveness that we introduced the coefficient $(-1)^{l}$ in $(20)$.

The idea for the estimator for $g_{22}$ is the solution to the equation

$E(z) \equiv \sum_{l m} W_{l} \zeta_{l m}(z)=0$

where the sum is taken over available values of $l$ and $m$, and the real positive quantity $W_{l}$ is an appropriate window function which one can choose at will. This will be a reasonable procedure to detect the statistical anisotropy if the statistical fluctuation of the quantity $E(z)$ for $z=g_{22}$ is sufficiently smaller than its statistical average for $z=0$ :

$\left\langle\left|E\left(g_{22}\right)\right|^{2}\right\rangle \ll|\langle E(0)\rangle|^{2}$,

or

$\left\langle\left|\sum_{l m} W_{l} \zeta_{l m}\left(g_{22}\right)\right|^{2}\right\rangle \ll\left|g_{22}\right|^{2}\left[\sum_{l m}(-1)^{l} W_{l} \xi_{l m}^{2} S_{l}\right]^{2}$.

An estimator for $g_{22}$ is then given by the solution to (23):

$\hat{g}_{22}=\frac{\sum_{l}(-1)^{l} l(l+1) W_{l} \sum_{m=2-l}^{l} a_{l m} a_{l, m-2}^{*}}{\sum_{l}(-1)^{l} l(l+1) \hat{C}_{l} W_{l} \sum_{m=2-l}^{l} \xi_{l m}^{2}}$.

This estimator is biased because its denominator is a random variable; but the biasing is small, since the denominator has a small relative dispersion $\sim 1 / \sqrt{N}$, where $N$ is the number of terms in (23). This last property is ensured by the coefficient $(-1)^{l}$ in the numerator and the denominator, making the denominator a sum of strictly positive terms. 


\section{Estimates of the Variance}

In estimating the variance on the left-hand side of (25), we can neglect the cross-correlation between terms with different values of $l$ in the sum, since this will produce terms of higher order in the small coefficients $g_{2 M}$. The number of such terms will be of the same order in $N$ as the number of leading terms, which is clear from the fact that there is no correlation between $a_{l m}$ and $a_{l^{\prime} m^{\prime}}$ with $\left|l-l^{\prime}\right|>2$ and $\left|m-m^{\prime}\right|>2$. The left-hand side of (25) is then estimated as

$$
\begin{aligned}
& \left\langle\left|\sum_{l m} W_{l} \zeta_{l m}\left(g_{22}\right)\right|^{2}\right\rangle \approx \\
& \approx \sum_{l} W_{l}^{2} \sum_{m=2-l}^{l} \sum_{n=2-l}^{l}\left\langle\zeta_{l m}\left(g_{22}\right) \zeta_{l n}^{*}\left(g_{22}\right)\right\rangle,
\end{aligned}
$$

up to terms $\mathcal{O}\left(g_{2 M}\right)$.

The expression for the correlation in (27) is given by

$$
\frac{\left\langle\zeta_{l m}\left(g_{22}\right) \zeta_{l n}^{*}\left(g_{22}\right)\right\rangle}{S_{l}^{2}}=\delta_{n m}+\delta_{n, 2-m}+\mathcal{O}\left(g_{2 M}\right) .
$$

Calculating the zero-order contribution to (27) in the quantities $g_{2 M}$, we have

$$
\begin{aligned}
& \sum_{l} W_{l}^{2} S_{l}^{2} \sum_{m n}^{\prime}\left(\delta_{n m}+\delta_{n, 2-m}\right)=2 \sum_{l} W_{l}^{2} S_{l}^{2} \sum_{m}^{\prime} 1= \\
& =2 \sum_{l}(2 l-1) W_{l} S_{l}^{2} \simeq 2 l_{\max }^{2} \overline{S_{l}^{2}}, \quad l_{\max } \gg 1,
\end{aligned}
$$

where the primed sums over $m$ and $n$ proceed from $2-l$ to $l$. Here,

$$
\overline{S_{l}^{2}}=\frac{\sum_{l} l W_{l} S_{l}^{2}}{\sum_{l} l W_{l}}, \quad l_{\max }=\left(2 \sum_{l} l W_{l}\right)^{1 / 2}
$$

are the characteristic quadratic average of $S_{l}$ and the effective maximal value of $l$, respectively.

The sum on the right-hand side of (25) is estimated as

$$
\begin{aligned}
& \sum_{l} W_{l} S_{l} \sum_{m}^{\prime}(-1)^{l} \xi_{l m}^{2} \simeq \frac{1}{2} \sqrt{\frac{5}{6 \pi}} \sum_{l} l W_{l} S_{l} \simeq \\
& \simeq \frac{1}{4} \sqrt{\frac{5}{6 \pi}} l_{\max }^{2} \overline{S_{l}}
\end{aligned}
$$

where $\overline{S_{l}}$ is defined similarly to (30). Criterion (25) then becomes

$2 \overline{S_{l}^{2}} \ll \frac{5}{96 \pi}\left|g_{22}\right|^{2} l_{\max }^{2}{\overline{S_{l}}}^{2} \lesssim \frac{5}{96 \pi}\left|g_{22}\right|^{2} l_{\max }^{2} \overline{S_{l}^{2}}$.

Thus, the magnitudes of $g_{22}$ that can be determined by this method are estimated as

$\left|g_{22}\right| \gtrsim \frac{11}{l_{\max }}$

For $l_{\max } \simeq 2000$ (hopefully available in the Planck experiment [17]), this gives $\left|g_{22}\right| \simeq 5.5 \times 10^{-3}$. With the data from the Wilkinson Microwave Anisotropy Probe [18], we have $l_{\max } \simeq 650$, and $\left|g_{22}\right| \simeq 1.7 \times 10^{-2}$. These crude estimates are in agreement with the estimates for the model-dependent minimum-variance estimators in [4], $\sigma_{g_{2 M}} \sim 3.8 \times 10^{-3}$ and $1.2 \times 10^{-2}$, respectively.

\section{Simulations}

To confirm our theoretical estimates, we perform numerical tests. For simplicity, the quantities $a_{l m}$ are generated independently for each $l$ with covariance determined by the correlation function (11) with prescribed values of $g_{2 M}$. For each independent simulation, we then compute the estimators $\hat{g}_{22}$ by (26). The results of simulations are presented in Figure. They confirm our theoretical expectations (33) and show a small bias of estimator (26).

\section{Discussion}

One of the robust predictions of inflation is the statistical isotropy of the Universe. It is of interest and importance to test this basic cosmological principle using modern astrophysical data. In doing this, one usually assumes some form of anisotropy in the primordial power spectrum. In this paper, we have constructed biased estimators for the scale-independent coefficients $g_{L M}$ of a simple statistically anisotropic primordial power spectrum in the form (2). They are similar to the minimumvariance estimators proposed in [4], but have the merit of being independent of the parameters of the underlying cosmological model, thus requiring no variation of these parameters in the observational tests of statistical anisotropy. We have determined the cosmic variance of our estimator for the coefficient $g_{22}$, which turned out to be comparable in magnitude to that presented in [4], allowing for a detection of the statistical anisotropy on the level of a few per cent [4]. We performed a number 

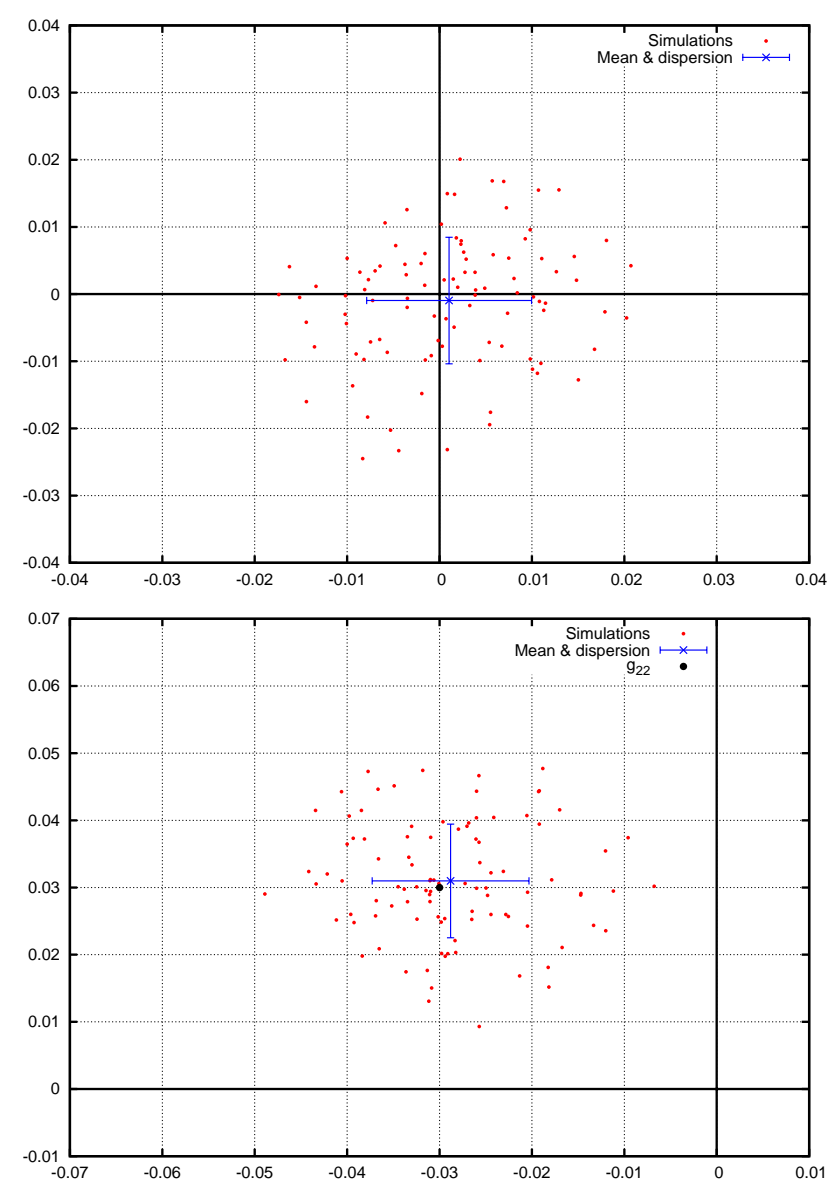

Results of simulations of 100 samples with zero (upper) and nonzero (lower) values of $g_{2 M}\left[g_{20}=0.01, g_{21}=0.02(1+i)\right.$, $\left.g_{22}=0.03(i-1)\right]$. The quantities $C_{l}$ in covariance (11) were set to unity. The points show the distribution of estimator (26) in the complex plane for $g_{22}$ with the window $W_{l}=1$ up to $l_{\max }=1000$. The grey color shows the mean and the dispersion separately for the real and imaginary parts of this distribution, and the black point in the lower figure indicates the value of $g_{22}=0.03(i-1)$ used in the generation of $a_{l m}$. One can see that the bias of estimator (26) is small, and the dispersion agrees with estimate (33)

of numerical simulations that confirmed our theoretical expectations.

In this paper, we did not consider the problem of noise, systematics, masks, and resolution which will introduce errors in the determination of the amplitudes $a_{l m}$ in (3). These issues relevant for the practical determination of the statistical anisotropy require further investigation.

This paper was supported by the Cosmomicrophysics programme and Program of Fundamental Research of the Division of Physics and Astronomy of the National Academy of Sciences of Ukraine.
1. A.D. Linde, Particle Physics and Inflationary Cosmology (Harwood, Chur, 1990).

2. P.G. Ferreira and J. Magueijo, Phys. Rev. D 56, 4578 (1997) [arXiv:astro-ph/9704052]; E.F. Bunn and D. Scott, MNRAS, 313, 331 (2000) [arXiv:astro$\mathrm{ph} / 9906044]$.

3. A. Hajian and T. Souradeep, Astrophys. J. 597, L5 (2003) [arXiv:astro-ph/0308001]; A. Hajian and T. Souradeep, arXiv:astro-ph/0501001; S. Basak, A. Hajian, and T. Souradeep, Phys. Rev. D 74, 021301 (2006) [arXiv:astro-ph/0603406]; T. Souradeep, A. Hajian, and S. Basak, New Astron. Rev. 50, 889 (2006) [arXiv:astroph/0607577].

4. A.R. Pullen and M. Kamionkowski, Phys. Rev. D 76, 103529 (2007) [arXiv:0709.1144 [astro-ph]].

5. S.M. Carroll, C.Y. Tseng, and M.B. Wise, Phys. Rev. D 81, 083501 (2010) [arXiv:0811.1086 [astro-ph]].

6. T.S. Pereira, C. Pitrou, and J.-P. Uzan, JCAP 0709, 006 (2007) [arXiv:0707.0736 [astro-ph]]; C. Pitrou, T.S. Pereira, and J.-P. Uzan, JCAP 0804, 004 (2008) [arXiv:0801.3596 [astro-ph]]; A.E. Gümrükçüoğlu, C.R. Contaldi, and M. Peloso, JCAP 0711, 005 (2007) [arXiv:0707.4179 [astro-ph]].

7. L. Ackerman, S.M. Carroll, and M.B. Wise, Phys. Rev. D 75, 083502 (2007) [Erratum-ibid. D 80, 069901 (2009)] [arXiv:astro-ph/0701357].

8. T.S. Koivisto and D.F. Mota, JCAP 0808, 021 (2008) [arXiv:0805.4229 [astro-ph]]; S. Yokoyama and J. Soda, JCAP 0808, 005 (2008) [arXiv:0805.4265 [astro-ph]]; S. Kanno, M. Kimura, J. Soda, and S. Yokoyama, JCAP 0808, 034 (2008) [arXiv:0806.2422 [hep-ph]]; M.A. Watanabe, S. Kanno, and J. Soda, Phys. Rev. Lett. 102, 191302 (2009) [arXiv:0902.2833 [hep-th]]; K. Dimopoulos, M. Karčiauskas, D.H. Lyth, and Y. Rodríguez, JCAP 0905, 013 (2009) [arXiv:0809.1055 [astro-ph]].

9. C.G. Böhmer and D.F. Mota, Phys. Lett. B 663, 168 (2008) [arXiv:0710.2003 [astro-ph]].

10. T. Koivisto and D.F. Mota, Astrophys. J. 679, 1 (2008) [arXiv:0707.0279 [astro-ph]].

11. C. Armendariz-Picon, JCAP 0709, 014 (2007) [arXiv:0705.1167 [astro-ph]]; M. Libanov and V. Rubakov, JCAP 1011, 045 (2010) [arXiv:1007.4949 [hep-th]]. M. Libanov, S. Ramazanov, and V. Rubakov, "Scalar perturbations in conformal rolling scenario with intermediate stage," arXiv:1102.1390 [hep-th].

12. E. Akofor, A.P. Balachandran, S.G. Jo, A. Joseph, and B.A. Qureshi, JHEP 0805, 092 (2008) [arXiv:0710.5897 [astro-ph]]; E. Akofor, A.P. Balachandran, A. Joseph, L. Pekowsky, and B.A. Qureshi, Phys. Rev. D 79, 063004 (2009) [arXiv:0806.2458 [astro-ph]].

13. N.E. Groeneboom, L. Ackerman, I.K. Wehus, and H.K. Eriksen, Astrophys. J. 722, 452 (2010) [arXiv:0911.0150 [astro-ph.CO]]. 
14. F.K. Hansen, A.J. Banday, K.M. Górski, H.K. Eriksen, and P.B. Lilje, Astrophys. J. 704, 1448 (2009) [arXiv:0812.3795 [astro-ph]]; H.K. Eriksen, F.K. Hansen, A.J. Banday, K.M. Górski, and P.B. Lilje, Astrophys. J. 605, 14 (2004) [Erratum-ibid. 609, 1198 (2004)] [arXiv:astro-ph/0307507]; J. Hoftuft, H.K. Eriksen, A.J. Banday, K.M. Górski, F.K. Hansen, and P.B. Lilje, Astrophys. J. 699, 985 (2009) [arXiv:0903.1229 [astroph.CO]].

15. L. Cayon, arXiv:1001.4680 [astro-ph.CO].

16. J.F. Donoghue, K. Dutta, and A. Ross, Phys. Rev. D 80, 023526 (2009) [arXiv:astro-ph/0703455]; A.L. Erickcek, M. Kamionkowski, and S.M. Carroll, Phys. Rev. D 78, 123520 (2008) [arXiv:0806.0377 [astro-ph]]; A.L. Erickcek, S.M. Carroll, and M. Kamionkowski, Phys. Rev. D 78, 083012 (2008) [arXiv:0808.1570 [astro-ph]].
17. http://www.rssd.esa.int/index.php?project=Planck 18. http://map.gsfc.nasa.gov

Received 01.03.11

МОДЕЛЬНО-НЕЗАЛЕЖНІ ОЦІНЮВАЧІ

ДЛЯ СТАТИСТИЧНОЇ АНІЗОТРОПІї КОСМІЧНОГО МІКРОХВИЛЬОВОГО ФОНУ

Ю.В. Штанов, Д.О. Савченко

$\mathrm{P}$ е з ю м е

Збудовано оцінювачі для тестування статистичної анізотропії космічного мікрохвильового фону, що виникає за рахунок квадрупольної масштабно-інваріантної анізотропії в первинному спектрі потужності. Оцінювачі не потребують знання основних космологічних параметрів. Визначено чутливість побудованих оцінювачів до величини статистичної анізотропії і проведено пробне числове моделювання, яке підтверджує зроблені теоретичні оцінки. 